\title{
Tensions dans la sécurité frontalière : entre sécurité étatique et insécurité économique locale
}

Tensions in border security: from state security to local economic insecurity Tensiones en la seguridad fronteriza: entre seguridad del Estado y inseguridad económica local

\section{Cléa Fortuné}

\section{(2) OpenEdition}

\section{Journals}

Édition électronique

URL : https://journals.openedition.org/ideas/11528

DOI : $10.4000 /$ ideas. 11528

ISSN : 1950-5701

Éditeur

Institut des Amériques

\section{Référence électronique}

Cléa Fortuné, «Tensions dans la sécurité frontalière : entre sécurité étatique et insécurité économique locale », IdeAs [En ligne], 18 | 2021, mis en ligne le 01 octobre 2021, consulté le 21 octobre 2021. URL: http://journals.openedition.org/ideas/11528 ; DOI : https://doi.org/10.4000/ideas.11528

Ce document a été généré automatiquement le 21 octobre 2021.

\section{c) (†)}

IdeAs - Idées d'Amériques est mis à disposition selon les termes de la licence Creative Commons Attribution - Pas d'Utilisation Commerciale - Pas de Modification 4.0 International. 


\section{Tensions dans la sécurité frontalière : entre sécurité étatique et insécurité économique locale}

Tensions in border security: from state security to local economic insecurity Tensiones en la seguridad fronteriza: entre seguridad del Estado y inseguridad económica local

\section{Cléa Fortuné}

\section{Introduction}

1 La frontière États-Unis/Mexique est la frontière la plus traversée au monde. Elle est caractérisée par les liens qui unissent ses villes jumelles de part et d'autre de la frontière. Les interactions entre ces dernières sont multiples: historiques, économiques, culturelles et sociales. Elles sont entretenues par les résidents frontaliers depuis que ces villes ont été créées dès la fin du xix siècle. En Arizona, des territoires historiquement espagnols, puis mexicains, sont devenus états-uniens avec l'Achat Gadsden en 1853. Mais même si les familles ont été séparées par la nouvelle frontière établie entre le Mexique et les États-Unis, les résidents ont conservé les liens avec l'autre côté, le pays voisin exerçant encore une grande influence sur les modes de vie frontaliers (Piñera Ramirez D., 1987 ; Langley L., 1987 ; Martínez O., 1994).

2 Toutefois, maintenir ces liens ne va pas de soi. En effet, la frontière ÉtatsUnis / Mexique est également caractérisée par des mesures sécuritaires, telles que des barrières ${ }^{1}$ séparant les deux pays, des outils technologiques qui permettent d'effectuer des contrôles sur les passages, des agents fédéraux comme la patrouille frontalière (Border Patrol, créée en 1924) et les agents du service des douanes (Customs and Border Protection), et des points d'entrée par lesquels les biens et les personnes transitent (Herzog L. et C. Sohn, 2019). Ces mesures sécuritaires sont mises en place dans des espaces transnationaux, marqués par des interactions quotidiennes entre résidents des 
villes mexicaines et états-uniennes. Comme le soulignent les géographes Victor Konrad et Heather N. Nicol :

The border and its adjacent spaces become places for sharing - a "transnational space", a zone of interaction [...] - a location where functional relationships are evident, and potentially an area of community integration (Konrad V. et H. Nicol, 2011).

3 Paradoxalement, cet espace transnational est donc à la fois le plus traversé au monde ${ }^{2}$ et l'un des plus sécurisés, notamment depuis les années 1990, années marquées par le processus simultané d'ouverture (debordering) et de fermeture (rebordering) des frontières (Albert M. et L. Brock, 1996 ; Andreas P. et T.J. Biersteker, 2003; Herzog L. et C. Sohn, 2019) ${ }^{3}$. Comment les résidents frontaliers réagissent-ils à ces mesures sécuritaires qui leur sont imposées ? Reflètent-elles l'état d'esprit des résidents frontaliers? Quelles sont leurs représentations de la sécurité ?

Cet article propose d'examiner le décalage qui existe entre ce qu'est la sécurité pour l'État fédéral et ce qu'est la sécurité pour les résidents frontaliers à partir d'une enquête de terrain menée entre 2017 et 2020 à Douglas (Arizona) et Agua Prieta (Sonora). Douglas est une petite ville de 15000 habitants, contrairement à sa ville mexicaine voisine qui en compte près de 200000 . Ces deux villes ont été créées au début $\mathrm{du} \mathrm{xx}^{\mathrm{e}}$ siècle et elles entretiennent entre elles des liens d'ordre économique, culturel et social. Elles constituent un binôme urbain qui n'a jusqu'ici été que très peu étudié. En effet, en études frontalières, les chercheurs se concentrent majoritairement sur les grandes villes frontalières comme El Paso/Ciudad Juarez, ou encore San Diego / Tijuana (Gutiérrez M. et al., 2021). Nous examinerons dans un premier temps la stratégie politique adoptée dès les années 1990 par l'État fédéral états-unien pour lutter contre les migrations irrégulières et pour sécuriser le territoire national. Nous utiliserons le concept de "sécuritisation ", c'est-à-dire la désignation d'une menace par une autorité légitime et acceptée comme telle par une audience significative (Buzan B. et al., 1997; Balzacq T., 2018), qui nous permettra de montrer comment l'État a construit un discours sécuritaire par lequel les migrations irrégulières sont devenues un problème de sécurité. Ce concept de "sécuritisation » comme processus politique mène à la sécurisation de la frontière, soit la mobilisation d'un ensemble de moyens financiers et humains pour mettre en œuvre une gamme de pratiques sécuritaires (Balzacq T., 2018). Dans un deuxième temps, nous montrerons que les mesures sécuritaires fédérales mises en place engendrent des formes d'insécurité pour les villes frontalières. Nous nous concentrerons en particulier sur la modification des modes de vie des résidents frontaliers, notamment leur mobilité, et sur les difficultés économiques engendrées par un ensemble de mesures fédérales. Enfin, nous porterons notre attention sur les représentations de la sécurité par les résidents frontaliers de Douglas. La sécurité, pour les résidents qui mènent une vie biculturelle ${ }^{4}$, est à l'opposé de ce qu'est la sécurité fédérale. Cet article se base sur des entretiens semi-directifs menés auprès de résidents de Douglas et d'Agua Prieta ${ }^{5}$. Parmi 95 enquêtés, 64 ont la possibilité de traverser la frontière car ils ont les documents nécessaires. Parmi eux, 52 traversent la frontière de façon régulière. Les interactions entre les deux côtés de la frontière font donc partie de leur vie quotidienne. C'est à partir de cette majorité de résidents frontaliers, que nous appellerons transnationaux ${ }^{6}$, que nous définirons ce que représente la sécurité pour eux. 


\section{La sécurité à la frontière États-Unis / Mexique}

5 Dans les années 1990, la frontière États-Unis / Mexique a connu un double phénomène d'ouverture et de fermeture des frontières, appelé défrontiérisation (debordering) et refrontiérisation (rebordering). Le processus de debordering, décrit comme la perméabilité grandissante des frontières (Albert M. et L. Brock, 1996), a été marqué par l'entrée en vigueur de l'ALENA (Accord de Libre-Échange Nord-Américain) en 1994, signé par le Canada, les États-Unis et le Mexique. Les frontières se sont ouvertes pour le mouvement des biens, des marchandises et des services, et la frontière ÉtatsUnis / Mexique est devenue l'un des points d'entrée les plus importants (Tableau 1).

Tableau : Passages entre la frontière États-Unis / Mexique suite à la signature de l'ALENA

\begin{tabular}{|l|l|}
\hline Années & Nombre de passages frontaliers entre les États-Unis et le Mexique \\
\hline 1996 & 276751448 \\
\hline 2000 & 392231869 \\
\hline 2005 & 336990567 \\
\hline 2010 & 242776894 \\
\hline 2015 & 267663436 \\
\hline 2020 & $171024678^{7}$ \\
\hline
\end{tabular}

Source : Bureau of Transportation Statistics, 2021b

6 L'ouverture des frontières a participé à remettre en cause la souveraineté de l'État fédéral sur son territoire national. Comme l'indique la politiste Wendy Brown, dans un contexte post-westphalien, l'État perd sa souveraineté au profit d'acteurs non étatiques et transnationaux (Brown W., 2010), ce qui le pousse à renforcer la sécurité à la frontière. Ce processus de refrontiérisation (rebordering) a pour but de contrôler la mobilité des personnes qui traversent la frontière, afin de réduire leurs mouvements tout en maintenant une économie sans frontière (Andreas P. et T.J. Biersteker, 2003).

7 Il faut noter que ce processus de rebordering a été mis en place pour répondre aux migrations irrégulières, suite à la fin du programme Bracero (un programme de travailleurs invités) en 1964 (Chavez L., 2011). Ce processus s'est poursuivi dans les années 1970 quand les États-Unis ont mené une guerre contre les drogues (Dunn T.J., 1996 ; Payan T., 2016). Dans les années 1990, les migrations irrégulières ${ }^{8}$ sont devenues source d'inquiétude pour l'opinion publique, en particulier en Californie. En effet, en 1994, la Californie connaissait une récession économique et un changement démographique ${ }^{9}$ (Douzet F., 2006) que le gouverneur Pete Wilson a utilisés pour se faire réélire. Il a profité des inquiétudes des citoyens au sujet de l'immigration et a accusé les immigrés d'être responsables des problèmes économiques de l'État (Massey D., 2010). Il a proposé un référendum pour voter en faveur de la Proposition 187, aussi appelée "SOS : Save Our State ", qui visait à refuser l'accès gratuit des immigrés aux services sociaux, aux services médicaux et à l'enseignement public. La frontière États- 
Unis / Mexique, considérée comme incontrôlable (Nevins J., 2010 : 4), est ainsi devenue le centre de l'attention politique et médiatique dans les années 1990, poussant l'État fédéral à s'emparer de la question de l'immigration (Cohen J., 2012) qui est passée d'un sujet de low politics à un sujet de high politics, à savoir un sujet vital pour la survie de l'État puisqu'il s'agit de sa sécurité nationale. Selon le géographe Joseph Nevins, la définition de la sécurité nationale fait partie des fonctions fondamentales de l'État moderne : « l'État moderne revendique le droit absolu de contrôler qui (et ce qui) entre ou sort de son territoire » (Nevins J., $2010: 30$ ).

Ainsi, en voulant réaffirmer sa souveraineté sur son territoire, l'État a construit un discours sécuritaire par lequel les migrations irrégulières sont devenues un problème de sécurité (Ceyhan A., 1998). Il s'agissait de protéger les citoyens contre des menaces extérieures (security). C'est ce que les chercheurs en Relations Internationales appellent la "sécuritisation", soit un processus ou une pratique discursive par lequel un problème politique, social ou public est traité comme un problème de sécurité (Balzacq T., 2018). Les immigrés ont ainsi fait l'objet de discours sécuritaires, en particulier les immigrés latinos, représentés comme une menace à la sécurité nationale des États-Unis (Chavez L., 2001 : 44). Les migrants ont été criminalisés sur le plan socioéconomique, identitaire, politique et sécuritaire (Ceyhan A. et A. Tsoukala, 2002) par des acteurs usant de stratégies discursives. On peut donner comme exemple la mise en scène de la peur de l'affaiblissement des contrôles aux frontières ainsi que la peur de l'État de perdre sa souveraineté (Simonneau D., 2020). Les autorités étatiques utilisent ainsi le mot frontière de façon répétée dans les discours sécuritaires pour faire porter l'attention publique sur une frontière considérée comme poreuse et synonyme d'insécurité. Par exemple, lors de sa présidence entre 2017 à 2021, Donald Trump a insisté de façon récurrente sur le lien entre migrants et criminels (Trump D., 2019). En 2019, dans son discours sur l'état de l'Union, il dénonçait le fait que "année après année, un nombre incalculable d'Américains sont tués par des criminels illégaux " (Paschal O. et al., 2019). En 2020, il faisait campagne avec le slogan «Build the Wall and Crime Will Fall ", insinuant qu'avec la construction d'une barrière, les migrants «illégaux» ne pourraient plus passer et ne pourraient plus tuer les États-Uniens (Trump D., 2019). Ces discours d'insécurisation visent à inquiéter la population (Bigo D., 2003) et permettent en retour de justifier la mise en place de moyens exceptionnels pour répondre aux menaces. On passe alors de la « sécuritisation » à la sécurisation de la frontière.

Traiter un enjeu comme un problème de sécurité amène l'État à mettre en place des moyens exceptionnels pour répondre aux menaces. Comme le souligne le politiste Peter Andreas :

Escalation has translated into tougher laws, rising budgets and agency growth, the deployment of more sophisticated equipment and surveillance technologies, and a growing fusion between law enforcement and national security institutions and missions. Of course, the most visible - and certainly most symbolic - sign of escalation has been the construction of more and bigger physical barriers. Escalation is also evident more generally in the sharper tone of the policy discourse about the border and the heightened prominence of "law and order" issues (Andreas P., $2009:$ 4).

La sécurisation de la frontière a commencé dès les années 1970 (Dunn T.J., 1996) et s'est renforcée dans les années 1990 sous l'administration Clinton - sous l'impulsion d'un Congrès à majorité républicaine - avec la mise en place des premières barrières en 
Californie et au Texas, faites de plateformes d'atterrissage pour hélicoptères, hautes de trois mètres et utilisées lors de la guerre du Vietnam (Hattam V., 2016). Plus de 1000 agents supplémentaires de la patrouille frontalière (Border Patrol) ont été déployés à la frontière États-Unis / Mexique pour contrôler les passages irréguliers (U.S. Border Patrol, 1994). Ces mesures faisaient partie de la stratégie appelée " prévention par la dissuasion» ("prevention through deterrence »). Mais plutôt que de décourager les candidats à l'immigration irrégulière, cette stratégie a créé un effet entonnoir (funnel effect) en les réorientant vers des zones dangereuses et désertiques, comme à Douglas en Arizona (Rubio Goldsmith R. et al., 2006) qui a vu sa première barrière installée en 1999 pour la séparer de sa ville voisine mexicaine Agua Prieta, alors une des zones de passage des migrations les plus importantes (Dillon S., 2000).

Depuis les années 1990, les mesures sécuritaires n'ont cessé d'être renforcées. Suite aux attentats terroristes du 11 septembre 2001, l'administration Bush a signé le Secure Fence Act et le Secure Border Initiative Network en 2006, permettant la création de davantage de barrières physiques, mais également la création d'une barrière virtuelle équipée d'outils technologiques de détection avancée, permettant de filtrer les «bonnes » et " mauvaises " migrations et qui serait la solution aux problèmes sociétaux que sont le terrorisme, les trafics de drogue et les migrations irrégulières (Heyman J., 2008 : 305). La construction de barrières séparant le Mexique des États-Unis a continué sous l'administration Obama avec l'installation de barrières secondaires qui ont agi comme un second palier sécuritaire derrière les premières barrières déjà en place, le déploiement de davantage d'agents de la Border Patrol et de nouveaux projets technologiques comme la Border Security Fencing Infrastructure and Technology (l'infrastructure et la technologie de la sécurité à la frontière) qui a accordé en 2014 un contrat de 145 millions de dollars à la société Elbit Systems of America pour la mise en place de tours intégrées et fixes, un outil technologique avancé qui permet de détecter les mouvements à plusieurs kilomètres. Avant que Donald Trump n'entre en fonction, 1000 kilomètres de barrières séparaient le Mexique des États-Unis sur plus de 3100 kilomètres de frontière. Mais dès son investiture en janvier 2017, il signait un décret présidentiel promettant de construire un « mur » physique et d'utiliser le matériel et la technologie appropriés pour contrôler la frontière le plus efficacement possible (White House, 2017). À la fin de son mandat, il s'est vanté d'avoir fait construire 800 kilomètres de barrières à la frontière États-Unis / Mexique. Bien que 643 kilomètres de barrières aient été des remplacements de barrières déjà existantes (Rodgers L. et D. Bailey, 2020), son administration a néanmoins construit des barrières plus hautes (passant de trois à neuf mètres de haut), notamment dans des zones reculées et protégées (Aguilera J., 2021), faisant fi des lois environnementales. La U.S. Customs and Border Protection (le service des douanes et de la protection des frontières des États-Unis) a célébré ce qu'il appelle le "système de barrières frontalières " ("border wall system ») en octobre 2020 (U.S. Customs and Border Protection, 2020). Selon Ken Cuccinelli - le secrétaire adjoint du Département de la Sécurité intérieure des États-Unis (Department of Homeland Security, DHS) entre 2019 et 2020 -, la construction de ces 800 kilomètres de barrières est «la preuve du leadership du Président Trump et de l'engagement de l'administration à sécuriser [la] frontière sud et à construire un système de barrières frontalières efficace qui protège tous les Américains" (U.S. Customs and Border Protection, 2020). Pour le DHS, la fonction du système de barrières frontalières est de stopper les migrants en situation irrégulière, d'assurer la sécurité nationale, et 
protéger les agents de la patrouille frontalière (U.S. Customs and Border Protection, 2020).

12 La réponse au sentiment de perte de souveraineté s'est donc traduite par la " sécuritisation » et la sécurisation, permettant à l'État de mettre en scène un pouvoir symbolique de contrôle des frontières (Brown W., 2010). En effet, ces barrières fonctionnent sur le plan symbolique et théâtral mais se révèlent inefficaces sur le plan fonctionnel. Le président Joe Biden, qui a pris ses fonctions en janvier 2021, a annoncé qu'il allait stopper la construction des barrières (sans pour autant les défaire) et qu'aucune barrière ne serait construite lors de son mandat. Cela constituerait une première depuis les années 1990. S'il admet que les barrières représentent une «perte d'argent ", il souhaite néanmoins renforcer la technologie à la frontière, notamment aux points d'entrée, principal point de passage des drogues (Biden J., 2020). En effet, si Joe Biden est opposé à la construction de barrières, il défend la mise en place de barrières virtuelles cachées et intelligentes en renforçant la frontière par une technologie de pointe (Miller T. et N. Buxton, 2020).

Les villes frontalières ont toujours été caractérisées par leurs liens historiques, économiques, et socio-culturels en raison de l'espace qu'elles partagent et qui les rend perméables aux influences des pays voisins. Elles sont également caractérisées par les résidents qui traversent la frontière très régulièrement, qui maintiennent et perpétuent ces liens. Mais le renforcement de la sécurité frontalière depuis les années 1990 a eu des conséquences sur ces liens, en affectant la mobilité des résidents et l'aspect économique au niveau local.

\section{L'insécurité engendrée par les mesures sécuritaires}

La frontière États-Unis / Mexique est caractérisée par le phénomène de "villes sœurs ", qui ont été créées vers la fin du XIX siècle, de façon simultanée, partageant ainsi un espace - la frontière - qui les rend perméables aux influences du pays voisin. Selon le géographe Michael Dear, pour les résidents frontaliers, «le territoire entre les deux nations n'est pas une question de souveraineté, de différence et de séparation, mais c'est plutôt le fondement de leur mode de vie » (Dear M., 2017). Ainsi, la position des villes à la frontière en fait des points d'entrée qui favorisent les échanges et le commerce. Espaces stratégiques pour l'économie, les points d'entrée à la frontière États-Unis / Mexique ont d'ailleurs augmenté de 10 à 50 entre les années 1990 et 2003 (Andreas P. et T.J. Biersteker, $2003: 6$ ). Chaque jour, il est estimé qu'une valeur de 1,7 milliards de dollars de biens et de services traversent la frontière par ces points d'entrée (Bureau of Transportation Statistics, 2021a; Schulz J.D., 2020). En plus des passages commerciaux, près de 665000 personnes traversent la frontière d'un pays à l'autre chaque jour, la première raison pour traverser étant le shopping, suivie du travail et des visites familiales (Cañas J. et al., 2006). Les consommateurs mexicains représentent une source de revenu essentielle pour les économies frontalières étatsuniennes, si bien que les élus frontaliers et les commerçants aménagent les espaces à la frontière pour attirer les consommateurs mexicains. Des centres commerciaux ont d'ailleurs été aménagés dans plusieurs de ces villes, à proximité du point d'entrée, accessible à pied pour les Mexicains qui ne traversent pas en voiture. C'est le cas par exemple à Calexico (Californie), à San Diego (Californie), ou encore à Douglas (Arizona). Longtemps marginalisées, les villes frontalières se sont tournées l'une vers l'autre pour 
leur développement (Ganster P. et D.E. Lorey, 2015 : 50), en particulier les petites villes frontalières comme Douglas, marginalisée à l'échelle des États-Unis et à l'échelle régionale transfrontalière (Fortuné $\mathrm{C}$., 2020). Ainsi, Douglas dépend de l'échelle locale transfrontalière pour son développement économique. Les résidents d'Agua Prieta qui ont les documents nécessaires pour passer la frontière représentent $80 \%$ de l'économie de Douglas, soit deux fois le budget annuel de la ville (City of Douglas, 2017). La population d'Agua Prieta augmentant rapidement, les résidents mexicains représentent un marché grandissant pour Douglas, qui compte treize fois moins d'habitants. La majorité des résidents d'Agua Prieta traversent donc pour aller faire leurs courses chez Walmart, à quelques minutes à pied de la frontière, pour acheter des produits qu'ils ne trouvent pas au Mexique ou qui sont moins chers comme le lait ou le chocolat en poudre (Fortuné C., 2020). L'impact économique de ces consommateurs mexicains est estimé à 740 millions de dollars chaque année sur la ville de Douglas (City of Douglas, 2017).

15 Toutefois, en imposant des mesures sécuritaires qui séparent les États-Unis du Mexique, l'État fédéral traite les résidents frontaliers mexicains comme des menaces, ce qui engendre une insécurité économique pour les villes frontalières états-uniennes qui dépendent des consommateurs mexicains pour leur survie. La mobilité des résidents mexicains est ainsi affectée par des contrôles plus poussés, notamment depuis les attentats du 11 septembre 2001, et par l'accès rendu plus difficile aux visas comme les Border Crossing Cards, un visa requis pour les Mexicains qui souhaitent venir aux ÉtatsUnis pour les loisirs. Ces mesures représentent un monopole de l'État dans le contrôle des personnes qui sont autorisées (ou non) à entrer aux États-Unis. C'est ce que souligne le sociologue Sergio Chávez :

Despite the arbitrariness in the process, BCCs and tourist visas [...] represent an attempt by the state to monopolize the legitimate means of movement by creating procedures and policies, establishing which types of persons may move within or cross their borders (Chávez S., $2016:$ 66).

16 Tous les résidents ne peuvent pas avoir accès à la Border Crossing Card, soit parce qu'elle est trop chère, soit parce qu'elle leur a été refusée lors de l'entretien avec les agents de la CBP. Pour les résidents détenteurs de ce visa, les contrôles étant plus poussés, les temps d'attente pour passer la frontière se sont allongés de quelques minutes à plusieurs heures aux heures de pointe. Les résidents ont donc dû adapter leur emploi du temps pour traverser la frontière, comme les élèves habitant à Agua Prieta mais allant à l'école à Douglas. C'est le cas de Rosie. Élève de lycée, elle se lève à $4 \mathrm{~h} 30$ le matin, pour se préparer, prendre le bus qui l'amène au point d'entrée, faire la queue pour passer les contrôles menés par les agents de la CBP, puis prendre un bus qui l'amène à l'école à Douglas et être à l'heure à $7 \mathrm{~h} 30$ (Fortuné C., 2020).

17 Les agents CBP qui assurent les contrôles aux points d'entrée sont les premiers ÉtatsUniens avec lesquelles les personnes qui entrent aux États-Unis ont un contact. Avant les années 1990, ces agents venaient des communautés frontalières et en faisant partie. Avec le renforcement sécuritaire, de plus en plus d'agents ont été déployés aux points d'entrée, avec pour conséquence qu'ils ne venaient plus forcément de la région frontalière et qu'ils n'en connaissaient pas nécessairement son fonctionnement. C'est ce qu'explique Del, résident mexicain-américain qui traverse la frontière entre Douglas et Agua Prieta plusieurs fois par semaines : 
And sometimes there are these real jerks there, they're new people, they don't understand the border, cultural competency is low. They're not familiar (entretien avec Del, Fortuné C., 2020). des conséquences sur la mobilité des résidents entre les deux pays. Des résidents mexicains et mexicains-américains évoquent des expériences de passages vécues comme stressantes. Ils évoquent avoir subi des discriminations en raison de leur couleur de peau, ils se sentent jugés par leurs déplacements entre les deux pays, alors même que la frontière est caractérisée par la mobilité des résidents frontaliers. Ceci amène ces résidents à changer leur fréquence de déplacement : ils traversent moins et les liens qui unissent les deux villes pourraient en pâtir à terme (Fortuné C., 2020).

La présidence de Donald Trump a renforcé l'impact sur la mobilité des résidents frontaliers (Beylier P.A. et C. Fortuné, 2020). Suite à ses propos tenus sur les Mexicains qu'il a décrits comme des "violeurs", des "criminels» et des "trafiquants de drogues », une baisse de passages entre le Mexique et les États-Unis a été observée pendant quelques mois. De janvier à février 2017, les passages entre Douglas et Agua Prieta sont tombés de 60000 à 40000 (Bureau of Transportation Statistics, 2021b). Selon le maire de Douglas de 2016 à 2020, Robert Uribe, les résidents mexicains ont été pris d'un sentiment de peur et d'anxiété, de confusion et d'incertitude qui les a incités à ne pas traverser durant cette période. Un autre facteur déterminant dans la diminution de la mobilité des résidents est la fermeture de la frontière aux voyageurs non essentiels, depuis mars 2020, suite à la déclaration d'urgence nationale de santé publique face à la Covid-19. À moins de détenir une Carte Verte ou un contrat de travail aux États-Unis, il n'est pas possible de venir aux États-Unis depuis le Mexique. Les consommateurs mexicains, qui viennent faire leurs courses en temps normal dans les villes frontalières états-uniennes, sont considérés comme des voyageurs non essentiels, ce qui a engendré des pertes de revenus considérables pour les commerces frontaliers états-uniens. À Nogales, de nombreux magasins ont déjà dû mettre la clé sous la porte, faute de clients (Janetsky M., 2021). Jusqu'en janvier 2021, les demandeurs d'asile étaient également considérés comme voyageurs non essentiels, et se sont vu refuser la prise en charge de leurs demandes d'asile aux États-Unis. Cela a fait suite au décret signé par Donald Trump pour suspendre l'immigration de façon temporaire afin d'éviter d'être «inondés» de cas de Covid-19 (Agren D., 2020). L'excuse sanitaire est donc venue renforcer les contrôles à la frontière.

Enfin, puisque les villes frontalières mexicaines se développent plus rapidement que leurs homologues états-uniennes, cela signifie que les villes du côté mexicain ouvrent de plus en plus de magasins :

The number of high-end stores in large Mexican cities is growing, giving their U.S. counterparts more competition. U.S. retailers still have the edge because they carry a greater variety of items, have the latest styles and often sell at significantly lower prices. These advantages may erode as Mexico's retail industry evolves (Cañas J. et al., 2006).

21 À terme, cela signifie que les consommateurs mexicains pourraient moins traverser vers les États-Unis, ce qui engendrerait des pertes de revenu considérables pour les villes frontalières états-uniennes. Or, les petites villes frontalières peinent à développer d'autres sources de revenus économiques. En raison des menaces que l'État définit comme étant les migrants en situation irrégulière et les trafiquants de drogue, ces villes souffrent de la réputation d'être dangereuses et peu accueillantes. C'est le cas de 
Douglas qui ne parvient pas à attirer d'investisseurs pour redynamiser son économie. En 2017, Douglas a fait une proposition à Amazon qui cherchait un nouveau centre de distribution. Amazon a refusé. Selon les anciens maires Danny Ortega (2012-2016) et Robert Uribe (2016-2020), c'est à cause des propos tenus par l'État fédéral sur les villes frontalières :

What's very challenging is that the rhetoric that plays out about border communities being dangerous, being scary, [doesn't make] investors really typically excited to invest in places like these (entretien avec Robert Uribe, Fortuné C., 2020).

Ces investisseurs sont d'autant moins enthousiastes lorsque les villes frontalières prennent des allures de zones de guerre, comme en novembre 2018 lorsque Donald Trump, souhaitant apporter une réponse symbolique face aux migrants de la caravane centraméricaine qui se déplaçaient vers les États-Unis pour y demander l'asile, a fait envoyer les militaires pour installer des fils barbelés le long de la barrière. Ainsi, la situation économique des petites villes frontalières peine à se développer, engendrant des taux de chômage et de pauvreté plus élevés qu'à l'échelle des États-Unis sur le temps long (Fortuné C., 2020).

En établissant des contrôles plus poussés à la frontière, la mobilité des résidents (et des migrants) s'en trouve affectée, ce qui engendre une insécurité d'ordre économique pour les petites villes frontalières états-uniennes. Les modes de vie binationaux des résidents sont remis en cause, d'autant plus avec la fermeture de la frontière pendant plus d'un an suite à la pandémie mondiale. Il existe donc un décalage entre le but des mesures sécuritaires (assurer la sécurité nationale) et les conséquences engendrées par celles-ci. Dans la troisième partie, nous analyserons ce qu'est la sécurité selon les résidents frontaliers transnationaux.

\section{Représentations de la sécurité pour les résidents frontaliers transnationaux}

Pour les élus locaux, la sécurité frontalière se traduit par le maintien des relations économiques binationales. Une des priorités pour la ville de Douglas est de relancer l'économie de la ville. Pour ce faire, les élus misent sur différentes stratégies. Tout d'abord, ils souhaitent moderniser le point d'entrée entre Douglas et Agua Prieta pour dynamiser l'économie des deux villes, mais aussi pour concurrencer d'autres villes frontalières comme Nogales (Arizona) / Nogales (Sonora). Les deux Nogales ont absorbé plus de 330000 camions par an à leur frontière entre 2016 et 2020, contre un peu plus de 25000 entre Agua Prieta et Douglas sur la même période, soit 13 fois moins qu'entre les deux Nogales (Fortuné C., 2020). Nogales absorbe plus de trafic notamment car elle est mieux connectée à Tucson, la deuxième plus grande ville d'Arizona, mais aussi parce qu'elle a un point d'entrée dédié spécifiquement aux camions, ce qui n'est pas le cas de Douglas. Après plusieurs demandes auprès de l'État fédéral pour moderniser son point d'entrée, Douglas a finalement reçu, en février 2019, l'autorisation et les fonds pour construire un point d'entrée destiné spécifiquement au passage des camions.

En plus de dynamiser le centre industriel derrière le point d'entrée où les consommateurs mexicains vont faire leurs courses, la municipalité de Douglas a voulu développer le centre historique, en en faisant un centre culturel et artistique pour de nouveau attirer les touristes du Mexique mais aussi des États-Unis. Suite à la construction du centre commercial accessible à pied depuis le point d'entrée, le centre 
historique où se trouvent les magasins locaux a en effet été déserté par les Mexicains. Faute de clients, de nombreux magasins ont dû fermer. D'où l'idée du maire Robert Uribe de faire du downtown un centre culturel et artistique binational afin de dynamiser la rue principale appelée $G$ Avenue. Rebaptisée la binational artwalk, cette avenue accueille des expositions d'art dans les magasins et dans la rue, des projections cinématographiques en plein air, et des festivals qui attirent les Mexicains d'Agua Prieta, comme les Fiestas Patrias, qui célèbrent l'indépendance du Mexique et qui permettent aux résidents de se réunir et de partager un moment festif au son de la musique norteña. C'est ce que souligne un résident de Douglas :

The last two [local] administrations have been trying to figure out how to enhance that idea of our connectedness. And they've gone on their way to cross the border a lot, and be part of what's going on in Agua Prieta and invite folks of Agua Prieta to be part of what's going on here in Douglas (entretien avec Mark, Fortuné C., 2020).

Pour attirer les États-Uniens, le maire Robert Uribe a fait installer un panneau publicitaire au sud de Tucson, promouvant la ville de Douglas comme ville de divertissement. Si Douglas attire déjà des États-Uniens qui viennent faire du tourisme médical à Agua Prieta (car le prix des médicaments et des consultations médicales est moins élevé du côté mexicain), cette activité n'est pas aussi développée que dans d'autres petites villes comme Los Algodones (Basse-Californie), qui compte 350 cabinets de dentistes, d'ophtalmologistes et de pharmacies pour une clientèle venant à $98 \%$ des États-Unis et du Canada (Yuma Arizona, 2020). La compétition entre corridors frontaliers a mené Douglas et Agua Prieta à passer des annonces dans des magazines pour retraités qui voudraient voyager pour un traitement médical ou pour s'approvisionner en produits pharmaceutiques. Il est mis en avant qu'il est possible pour eux de se loger du côté états-unien (ce qui les rassure), et de se rendre chez le médecin, le dentiste ou l'ophtalmologiste du côté mexicain pour se faire soigner.

Au-delà de la municipalité, les résidents eux-mêmes se mobilisent pour redynamiser leur ville. Un projet monté par un groupe de résidents de Douglas était d'ouvrir au public le tunnel d'El Chapo Guzman. En effet, le premier tunnel de ce baron de la drogue a été découvert à Douglas en 1990. Le projet visait à jouer sur l'image de la ville frontalière considérée comme zone de passage de drogues afin d'attirer les touristes états-uniens, qui souvent s'arrêtent à Bisbee, à 30 minutes au nord de Douglas. Le projet a jusqu'ici été refusé, une des raisons étant que le tunnel se trouve dans un bâtiment appartenant à la patrouille frontalière qui devrait donner son accord et l'aménager pour les touristes. D'autres actions des résidents consistent à s'assurer eux-mêmes du bon accueil de leurs voisins mexicains à travers l'organisation de Welcoming Actions, des actions de bienvenue. Les résidents se mettent les uns derrière les autres le long de la route derrière le point d'entrée pour accueillir les personnes qui passent du Mexique aux États-Unis avec des panneaux affichant des messages de bienvenue, comme "Welcome! Construyemos puentes, no muros» («Bienvenue, nous construisons des ponts, pas des murs) ou encore «Bienvenidos todos, Mexicanos, Centro Americanos, todos nuestros vecinos» (Bienvenue à tous, Mexicains, Centraméricains, tous nos voisins»). Ces actions ont été organisées par un résident de Douglas, James Martin, lors de l'élection de Donald Trump en novembre 2016, et lors des renforcements sécuritaires en novembre 2018 lorsque les militaires installaient les fils barbelés le long de la barrière (Fortuné $C$., 2020). Pour les résidents frontaliers, se sentir en sécurité signifie maintenir les relations binationales avec le Mexique, au sens de safety (Lee Maril R., 2011). 
Un autre aspect crucial pour les résidents frontaliers transnationaux qui entretiennent des liens avec le Mexique, est de traiter les causes fondamentales des migrations et donc de permettre aux individus de ne pas avoir à migrer. Ils souhaitent agir sur les causes des migrations plutôt que sur les conséquences. Il existe une organisation humanitaire presbytérienne transfrontalière, présente à Douglas et Agua Prieta, appelée Frontera de Cristo qui incarne l'expression connue à la frontière, " construisons des ponts plutôt que des murs ». Quand son coordinateur, Mark Adams, est arrivé à Agua Prieta depuis la Caroline du Sud en 1998, il s'est aperçu que de nombreux résidents d'Agua Prieta venaient du Chiapas, un des États les plus pauvres du Mexique et pourtant l'un des plus grands producteurs de café. Le travail dans les champs de café ne rapportant que peu d'argent, les résidents avaient migré au nord du Mexique dans l'espoir de traverser aux États-Unis ou de travailler dans les maquiladoras, des usines de montage qui se sont multipliées le long de la frontière nord du Mexique et qui bénéficient d'exonérations fiscales pour importer des pièces qui y seront assemblées et intégralement réexportées (Villavicencio D., 2004). Les salaires y sont plus élevés que dans les champs de café. En 2002, Mark Adams a décidé d'aller rencontrer ces caféiculteurs du Chiapas pour entendre leur histoire et leur proposer de créer une coopérative dont les bénéfices iraient aux caféiculteurs, ce qui leur permettrait de subvenir à leurs besoins et à ceux de leur famille. L'initiative qui en a découlé est Café Justo/Just Coffee. Elle fonctionne sur le principe suivant: les caféiculteurs de Café Justo vendent leurs grains de café à Frontera de Cristo plutôt qu'à des intermédiaires. Frontera de Cristo se charge de transporter les grains jusqu'à Agua Prieta où ils sont torréfiés, mis en paquet puis vendus aux États-Unis et au Canada. Les bénéfices des ventes de café reviennent aux caféiculteurs, ce qui leur permet de subvenir aux besoins de leur famille et de rester au Chiapas. Edmundo fait partie de la coopérative, à Salvador Urbina, au Chiapas. Avant de faire partie de la coopérative, il a migré aux États-Unis, à Atlanta, en Géorgie, où il travaillait dans un club de golf, laissant sa femme et ses deux jeunes filles derrière lui, afin de leur envoyer de l'argent. Lorsqu'il est rentré à Salvador Urbina pour leur rendre visite, il avait pour but de repartir quelques mois plus tard. Mais quand il a entendu parler de la coopérative par ses collègues caféiculteurs, il a décidé de s'intégrer au groupe et de faire fonctionner la coopérative. Aujourd'hui, grâce à celle-ci, Edmundo et sa famille ont pu souscrire une assurance santé qu'ils n'avaient pas les moyens de payer jusque-là. Ils ont également pu envoyer leurs filles à Tapachula, au Chiapas, suivre des études pour devenir infirmières, ce dont ils sont très fiers. Cette coopérative a été une telle réussite que les caféiculteurs et Frontera de Cristo ont ouvert le café Café Justo à Agua Prieta en 2015, surnommé « le Starbucks local », qui fait fonctionner l'économie de la ville frontalière en y employant des résidents locaux et d'anciens toxicomanes en réinsertion (Fortuné C., 2020).

\section{Conclusion}

Les politiques de sécurité à la frontière États-Unis / Mexique sont ambivalentes, à la fois facteurs de réassurance et facteurs d'insécurité. La sécurité (security), pour l'État fédéral états-unien, est de lutter contre une menace, définie comme étant les migrants en situation irrégulière et associés aux trafiquants de drogue et aux criminels. Cette "sécuritisation", soit la désignation d'une menace comme un problème de sécurité, 
justifie, selon l'État, la mise en place de moyens exceptionnels pour assurer la sécurité nationale, ce qui passe par un renforcement sécuritaire (barrières, outils technologiques et déploiement d'agents frontaliers) qui n'a eu de cesse de se renforcer depuis les années 1990. Toutefois, ces mesures sécuritaires perturbent les modes de vie binationaux, caractéristiques des villes frontalières. Faisant face à des pratiques discriminatoires et à des contrôles plus poussés, certains résidents, notamment mexicain-américains et mexicains, réduisent leurs déplacements d'un pays à l'autre. Cette modification de la mobilité des résidents frontaliers engendre à son tour une insécurité d'ordre économique pour les petites villes frontalières états-uniennes. On passe de la sécurité au sens de security - le gouvernement souhaite protéger son territoire national contre des menaces extérieures - à la sécurité au sens de safety (Lee Maril R., 2011) - les résidents frontaliers souhaitent maintenir leur vie binationale, menacée par la sécurité imposée par l'État. L'État lui-même devient alors une menace à la sécurité (safety) des habitants de la région frontalière. Cet article n'a mentionné que l'insécurité économique, mais d'autres insécurités sont engendrées par les mesures sécuritaires fédérales. Nous pouvons citer une insécurité environnementale de par la construction de barrières dans des zones protégées (Fortuné C., 2020), et une insécurité humaine car plus les mesures sécuritaires se développent, et plus les migrants sont vulnérables aux organisations criminelles transnationales (Slack J., 2019).

Si la sécurité frontalière a du sens aux yeux de l'État fédéral de par son pouvoir symbolique (Andreas P., 2009 : 8), elle revêt une autre dimension pour une majorité de résidents frontaliers transnationaux. Pour ces derniers, il existe un décalage entre ce qu'est la sécurité imposée par Washington, et ce qu'est la sécurité frontalière à l'échelle locale. Une réelle sécurité frontalière passe par la préservation de leur vie binationale, le maintien des relations économiques binationales et par une sécurité humaine qui traite les causes des migrations plutôt que leurs conséquences. La fermeture de la frontière suite à la crise sanitaire de la Covid-19 a accentué ces tensions entre mesures de sécurité fédérales et perceptions locales.

\section{BIBLIOGRAPHIE}

Agren, David, « "Mexico has become Trump's wall": how AMLO became an immigration enforcer ", The Guardian, 26 janvier 2020, https://www.theguardian.com/world/2020/jan/26/mexicoimmigration-amlo-enforcement-trump, page consultée le 20 août 2021.

Aguilera, Jasmine, « What to Know About The Legacy of Trump's Border Wall », Time, 12 janvier 2021, https://time.com/5928808/trump-border-wall-what-to-know/, page consultée le 13 mars 2021.

Albert, Mathias et Brock, Lothar, « Debordering the world of states: New spaces in international relations ", New Political Science, vol. 18, n 1, 1996, p. 69-106.

Andreas, Peter, Border Games: Policing the U.S.-Mexico Divide, Ithaca, Cornell University Press, 2009. 
Andreas, Peter, Biersteker, Thomas J., The Rebordering of North America: Integration and Exclusion in a New Security Context, New York, Routledge, 2003.

Balzacq, Thierry, « Théories de la sécuritisation, 1989-2018 », Études internationales, vol. 49, n 1, 2018, p. 7-24.

Beylier, Pierre-Alexandre et Fortuné, Cléa, « Cross-Border Mobility in Nogales Since Trump's Election », Journal of Borderlands Studies, 2020, p. 1-22.

Biden, Joseph, « The Biden Plan for Securing Our Values as a Nation of Immigrants ", Joe Biden for President: Official Campaign Website, 2020, https://joebiden.com/immigration/, page consultée le 13 mars 2021.

Bigo, Didier, « Comment douter de la sécurité ? », Hommes \& Migrations, n 1241, 2003, p. 32-42.

Brown, Wendy, Walled States, Waning Sovereignty, Cambridge, MIT Press, 2010.

Bureau of Transportation Statistics, « Overview of U.S.-North American Freight by Port, Commodity Group, and Mode », Bureau of Transportation Statistics, 2021a, https://explore.dot.gov/ t/BTS/views/Dashboard_PortbyCommodity/Overview?

\%3Aiid=1\&\%3AisGuestRedirectFromVizportal=y\&\%3Aembed=y\&\%3AusingOldHashUrl=true, page consultée le 13 mars 2021.

Bureau of Transportation Statistics, «Classeur : Border Crossing Data », Bureau of Transportation Statistics, 2021b., https://explore.dot.gov/views/BorderCrossingData/

Annual?:isGuestRedirectFromVizportal=y\&:embed=y, page consultée le 13 mars 2021.

Buzan, Barry, Weaver, Ole et De Wilde, Jaap, Security: A New Framework for Analysis, Boulder, Colorado, Lynne Rienner Publishers Inc., 1997.

Cañas, Jesus, Coronado, Roberto et Phillips, Keith, « Border Benefits from Mexican Shoppers », Southwest economy, mai / juin 2006.

Ceyhan, Ayse, « Analyser la sécurité : Dillon, Waever, Williams et les autres », Cultures \& Conflits, vol. 31-31, n 3, 1998, p. 0-0.

Ceyhan, Ayse et Tsoukala, Anastassia, « The Securitization of Migration in Western Societies: Ambivalent Discourses and Policies », Alternatives, vol. 27, Special Issue, 2002, p. 21-39.

Chavez, Leo. R., Covering Immigration - Popular Images \& the Politics of the Nation. Berkeley, University of California Press, 2001.

Chávez, Sergio, Border Lives: Fronterizos, Transnational Migrants, and Commuters in Tijuana, New York, Oxford University Press, 2016.

City of Douglas, Douglas Arizona 2017 State of the City, 2017.

Cohen, James, À la poursuite des illégaux : Politiques et mouvements anti-immigrés aux États-Unis, Bellecombe-en-Bauges, Éditions du Croquant, 2012.

Dear, Michael, « Americans and Mexicans Living at the Border Are More Connected than Divided », The Conversation, 6 mars 2017, http://theconversation.com/americans-and-mexicansliving-at-the-border-are-more- connected-than-divided-72348, page consultée le 20 août 2020.

Dillon, Sam, « Agua Prieta Journal; Boom Turns Border to Speed Bump », The New York Times, 18 janvier 2000, https://www.nytimes.com/2000/01/18/world/agua-prieta-journal-boom-turnsborder-to-speed-bump.html, page consultée le 13 mars 2021.

Douzet, Frédérick, « Ségrégation et balkanisation politique de la Californie », Hérodote, vol. 3, $n^{\circ} 122,2006$, p. 66-91. 
Dunn, Timothy J., The Militarization of the U.S.-Mexico Border, 1978-1992, Austin, University of Texas Press, 1996.

Fortuné, Cléa, Sécurité frontalière, insécurité locale dans les borderlands États-Unis/Mexique. Étude de Douglas (Arizona) et Agua Prieta (Sonora), Thèse de civilisation américaine, Sorbonne Nouvelle, 2020.

Ganster, Paul et Lorey, David E., The U.S.-Mexican Border Today: Conflict and Cooperation in Historical Perspective, Lanham, Rowman \& Littlefield Publishers, 2015.

Gutiérrez, Manuel, Sánchez-Atondo, Alejandro et García, Leonel et al., « Understanding crossborder mobility in medium-small Mexico-U.S. Binational regions. Mexicali-Imperial Valley case study », Transportation Research Interdisciplinary Perspectives, vol. 9, 2021.

Hattam, Vicotria, «Imperial designs: Remembering Vietnam at the US-Mexico border wall », Memory Studies, vol. 9, n 1, 2016, p. 27-47.

Herzog, Lawrence A. et Sohn, Christophe, « The co-mingling of bordering dynamics in the San Diego-Tijuana cross-border metropolis », Territory, Politics, Governance, vol. 7, n², 2019, p. 177-199.

Heyman, Josiah McC, « Constructing a Virtual Wall: Race and Citizenship in U.S.-Mexico Border Policing ", Journal of the Southwest, vol. 50, n 3, 2008, p. 305-333.

Janetsky, Megan, «In US-Mexico border towns, pandemic hits businesses especially hard », Christian Science Monitor, 5 janvier 2021, https://www.csmonitor.com/Business/2021/0105/In-USMexico-border-towns-pandemic-hits-businesses-especially-hard, page consultée le 13 mars 2021.

Konrad, Victor et Nicol, Heather N., « Border Culture, the Boundary Between Canada and the United States of America, and the Advancement of Borderlands Theory », Geopolitics, vol. 16, $\mathrm{n}^{\circ} 1$, 2011, p. 70-90.

Langley, Lester, Mexamerica: Two Countries, One Future, New York, Crown, 1987.

Lee Maril, Robert, The Fence: National Security, Public Safety, and Illegal Immigration Along the U.S.Mexico Border, Lubbock, Texas University Press, 2011.

Martínez, Oscar, Border People: Life and Society in the US-Mexico Borderlands, Tucson, University of Arizona Press, 1994.

Massey, Douglas. S. (Éd.), New Faces in New Places: The Changing Geography of American Immigration, New York, Russell Sage Foundation, 2010.

Miller, Todd et Nick Buxton, « Biden's Border: The industry, the Democrats and the 2020 elections », TNI, février 2021, https://www.tni.org/en/bidensborder, page consultée le 19 août 2021.

Nevins, Joseph, Operation Gatekeeper and Beyond: The War On «Illegals » and the Remaking of the U.S. Mexico Boundary, New York, Routledge, 2010.

Paschal, Olivia et Carlisle, Madeleine, « Read President Trump's Second State of the Union Address ", The Atlantic, 5 février 2019, https://www.theatlantic.com/politics/archive/2019/02/ trumps-second-state-union-full-text/582025/, page consultée le 13 mars 2021.

Payan, Tony, The Three U.S.-Mexico Border Wars: Drugs, Immigration, and Homeland Security, Santa Barbara, ABC-CLIO, 2016.

Piñera Ramirez, David, Visión histórica de la frontera norte de México, Mexicali, Universidad Autónoma de Baja California, Centro de Investigactiones Históricas UNAM-UABC, 1987. 
Rodgers, Lucy et Bailey, Dominic, « Trump wall: How much has he actually built? », BBC News, 31 octobre 2020, https://www.bbc.com/news/world-us-canada-46824649, page consultée le 13 mars 2021.

Rubio Goldsmith, Raquel, McCormick, Melissa et Martinez, Daniel et al., « The "Funnel Effect" \& Recovered Bodies of Unauthorized Migrants Processed by the Pima County Office of the Medical Examiner, 1990-2005 ", SSRN, 1 octobre 2006, https://papers.ssrn.com/sol3/papers.cfm? abstract_id=3040107, page consultée le 20 août 2021.

Schulz, John D., « 2020 Cross-border Update: Even more confusion ahead », Logistics Management, 3 juin 2020, https://www.logisticsmgmt.com/article/

2020_cross_border_update_even_more_confusion_ahead, page consultée le 13 mars 2021.

Simonneau, Damien, L'Obsession du Mur. Politique de militarisation des frontières en Israël et aux ÉtatsUnis, Berne, Peter Lang, 2020.

Slack, Jeremy, Deported to Death: How Drug Violence Is Changing Migration on the US-Mexico Border, Oakland, University of California Press, 2019.

Trump, Donald, Donald J. Trump sur Twitter : "BUILD A WALL and CRIME WILL FALL! This is the new theme, for two years until the Wall is finished (under construction now), of the Republican Party. Use it and pray!", Twitter, 23 janvier 2019, https://twitter.com/realDonaldTrump/status/ 1088058134713823233 , page consultée le 13 mars 2021.

U.S. Border Patrol, « Border Patrol Strategic Plan 1994 and Beyond », Homeland Security Digital Library, juillet 1994, https://www.hsdl.org/?abstract\&did=721845, page consultée le 19 août 2021.

U.S. Customs and Border Protection, « DHS and CBP Celebrate 400 Miles of New Border Wall System », U.S. Customs and Border Protection, 29 octobre 2021, https://www.cbp.gov/newsroom/ national-media-release/dhs-and-cbp-celebrate-400-miles-new-border-wall-system, page consultée le 13 mars 2021.

Villavicencio, Daniel, « Les "Maquiladoras" de la frontière nord du Mexique et la création de réseaux binationaux d'innovation », Innovations, vol. 19, n 1, 2004, p. 143-161.

White House, « Executive Order : Border Security and Immigration Enforcement Improvements ", The White House, janvier 2017, https://www.whitehouse.gov/presidential-actions/executiveorder-border-security-immigration-enforcement-improvements/, page consultée le 13 mars 2021.

Yuma Arizona, «Going to Mexico - Welcome To Yuma, Arizona - On The River's Edge », Visit Yuma, 2020, https://www.visityuma.com/going-to-mexico.html, page consultée le 20 août 2020.

\section{NOTES}

1. Le terme "mur » (wall) est régulièrement utilisé par les résidents frontaliers, mais nous préférons utiliser le terme «barrières » qui, selon nous, permet de rendre compte de la diversité de démarcations visuelles qui séparent le Mexique des États-Unis.

2. En 2018, environ 300 millions d'individus ont passé la frontière entre les États-Unis et le Mexique selon le Bureau of Transportation Statistics (Bureau of Transportation Statistics, 2021b).

3. Ces termes sont définis plus bas.

4. Selon Oscar Martínez, les résidents qui mènent une vie biculturelle apprécient les différences culturelles. Ils voient d'un bon œil le bilinguisme et le biculturalisme (Martínez O., 1994 : 132). 
5. Les observations participantes et entretiens semi-directifs ont été menés entre 2017 et 2020 des deux côtés de la frontière, en anglais et en espagnol. Une aide financière a été apportée par l'ED 625 MAGIIE de l'Université Sorbonne Nouvelle et par la commission franco-américaine Fulbright.

6. Nous reprenons ce terme de la typologie établie par Oscar Martínez dans son ouvrage Border People: Life and Society in the US-Mexico Borderlands, publié en 1994. Il définit les résidents transnationaux comme des individus qui maintiennent des liens importants avec le pays voisin et dont le mode de vie reflète des influences étrangères (Martínez O., 1994).

7. La baisse du nombre de passages en 2020 s'explique par la fermeture de la frontière suite à la crise sanitaire de la Covid-19.

8. Dans les textes officiels du Department of Homeland Security, le terme «illegal alien » est utilisé pour faire référence aux immigrés qui entrent sur le territoire états-unien en enfreignant la loi et qui sont donc considérés comme commettant une infraction. Nous préférons ici le terme de migrations irrégulières, plus neutre selon nous.

9. Selon Frédérick Douzet, « après les lois de libéralisation de 1965, qui ont pris effet en 1968, l'immigration a progressivement augmenté aux États-Unis puis s'est considérablement accélérée dans les années 1980 et 1990 . Or, la Californie, par sa situation géographique, s'est trouvée le premier destinataire de cette immigration en provenance d'Amérique latine et centrale (40\%) et d'Asie (35\%), une tendance renforcée par une immigration clandestine essentiellement mexicaine (plus des deux tiers) » (Douzet F., 2006).

\section{RÉSUMÉS}

La frontière États-Unis / Mexique fait l'objet de mesures sécuritaires depuis les années 1970, renforcées dans les années 1990. La ville frontalière de Douglas en Arizona ne fait pas exception. Dans le but de stopper les migrations et le trafic de drogue, une barrière a été construite pour séparer Douglas de sa ville voisine mexicaine Agua Prieta (Sonora), remettant en cause les liens transfrontaliers. Cet article se concentre sur les résidents de ces deux villes et sur leurs représentations de la sécurité. Les barrières imposées par le gouvernement fédéral reflètent-telles l'état d'esprit local ? Quel est l'impact de l'appareil de sécurisation à la frontière sur les habitants et sur la coopération historique transfrontalière? Les mesures sécuritaires n'engendrent-elles pas des formes d'insécurité ? Nous apporterons une analyse de la définition de la sécurité perçue par les habitants grâce aux enquêtes de terrain réalisées à Douglas et à Agua Prieta entre 2017 et 2020 pour comprendre les tensions - renforcées par la pandémie de COVID 19 - entre les enjeux de politique intérieure à l'échelle nationale et les enjeux locaux perçus par les résidents frontaliers. Pour ces derniers, la sécurité frontalière passe par la préservation de leur vie binationale, le maintien des relations économiques binationales et une sécurité humaine qui traite les causes des migrations.

Security concerns at the U.S./Mexico border started in the 1970s and have been reinforced on a continual basis since the 1990s. The border town of Douglas, Arizona, is no exception. At the end of the 1990s, a barrier was built to separate Douglas from its Mexican sister city, Agua Prieta, Sonora. If the aim was to stop irregular migrations and drug trafficking, it actually resulted in disturbing the transborder connectedness and the border residents' way of life. This article will thus focus on the border residents' representation of security. Are the security measures 
accepted by the border residents? What is the impact of these security measures on the historical transborder cooperation? Do security measures bring insecurity? Thanks to interviews conducted in Douglas and Agua Prieta between 2017 and 2020, we will analyze the definitions that border residents give to border security, enhancing the tensions - that have been reinforced by COVID-19 - between the federal definition and the local definition of security. For border residents, border security means preserving their binational life, preserving the binational economic ties and preserving human security by focusing on the roots of migration.

Las medidas de seguridad entre la frontera entre los Estados Unidos y México empezaron en los años 1970 y han intensificado desde los años 1990. La ciudad fronteriza de Douglas, Arizona, no constituye una excepción. A finales de los años 1990, una barrera se construyó, separando Douglas de su ciudad vecina mexicana Agua Prieta, Sonora, con el fin de luchar contra la migración irregular y el narcotráfico. Pero la consecuencia fue de perturbar las conexiones entre los dos comunidades y la forma de vivir de los fronterizos. Este articulo se refiere a la representación de los fronterizos sobre la seguridad. ¿Son las medidas de seguridad aceptadas en las ciudades fronterizas? ¿Cuáles son los impactos sobre las relaciones transfronterizas históricas? ¿Provocan formas de inseguridad? Por medio de entrevistas con informantes en Douglas y Agua Prieta entre 2017 y 2020, este artículo va a analizar las definiciones de seguridad dado por los fronterizos. Hay tensiones - fortalecidas por la crisis de COVID-19 - entre la seguridad definida por el estado federal y la seguridad definida por los residentes de Douglas y Agua Prieta. Para ellos, la seguridad fronteriza significa mantener una vida binacional, mantener las relaciones económicas binacionales y una seguridad humana que trata las causas de las migraciones.

INDEX

Keywords : Security, insecurity, barrier, border, U.S. / Mexico

Palabras claves : Seguridad, inseguridad, barrera, frontera, EEUU / México

Mots-clés : Sécurité, insécurité, barrière, frontière, États-Unis / Mexique

\section{AUTEUR}

\section{CLÉA FORTUNÉ}

Cléa Fortuné est ATER à l'Université Grenoble Alpes. Elle est docteure en civilisation américaine de l'Université Sorbonne Nouvelle et membre du Center for Research on the English-speaking World (CREW EA 4399). clea.fortune@gmail.com 